\title{
Thyroid Abnormalities in Correlation with Stage of CKD in Tribal Population of Chhattisgarh
}

\author{
Priya Banjare \\ B.SC Dialysis Technology, Pt. J.N.M. Medical College, Raipur, Chhattisgarh, India
}

\begin{abstract}
Chronic Kidney Disease is a worldwide health problem with an increasing incidence and prevalence. Abnormalities in the structure and function of the thyroid gland and in the metabolism and plasma concentration of thyroid hormones are common in patients with CKD. In view of variability of thyroid profile in CKD patients in previous studies, a prospective study of various thyroid function has been undertaken to establish a correlation if any between thyroid dysfunction and severity of renal diseases. Total number of 50 patients with Chronic Kidney Disease on conservative management fulfilling the criteria for CKD who were admitted in Department of Medicine under nephrology unit, Dr. Bhimrao Ambedkar Hospital Raipur (C.G.), during the period of February 2018-March 2018 were selected in this prospective study. The result showed that out of the 50 patients with CKD 29 patients had low T3 syndrome $(0.2-2.0 \mathrm{ng} / \mathrm{ml}$, mean 0.67$)$ which accounts for $58 \%$ of the patients, 12 patients had low T4 syndrome $(0.5-8.5 \mu \mathrm{g} / \mathrm{ml}$, mean 5.65$)$ which accounts for $24 \%$ of the patients and 4 patients had primary hypothyroidism TSH $>20 \mu \mathrm{IU} / \mathrm{ml}$. Excluding Primary Hypothyroidism, analysis of serum T3, T4 and TSH in the study subjects shows very high significance, $\mathrm{p}<0.001$. Thyroid Dysfunction occurred in $66 \%$ of the patients with chronic kidney disease in our study, it does not indicate a state of hypothyroidism, but a reflection of the state of chronic illness/malnutrition. The low T3 state of CKD can be viewed as being protective, promoting conservation of protein. The number of patients with low T3 syndrome progressively increases with the severity of renal failure.
\end{abstract}

\section{Keywords: chronic kidney disease, low T3syndrome, Thyroid dysfunction}

\section{Materials and Methods}

Patients who were admitted in the department of medicine under nephrology unit, Dr. Bhimrao Ambedkar Hospital Raipur (C.G.) between month of February to March 2018. The present study is conducted on total 60 patients, who were diagnose to have Chronic Kidney Disease. Ethical clearance has taken from the ethical committee of the Pt. JNM Medical College Raipur, prior to conduct the study. All participants and family members of the patients were provided written information consent. An approved prestructured tools or format was used to collect the information that include basic parameters age, sex, pulse, blood pressure, Peripheral smear for anaemia, blood urea, Serum Creatinine, Creatinine clearance (using Cockcroft Gault formula) ,Serum electrolytes ,Serum calcium, phosphorous and uric acid, Serum cholesterol, 24 hours urinary protein, Serum protein (Total protein / albumin / globulin),USG abdomen for evidence of chronic kidney disease .After selection of patients, fulfilling the above criteria, about $5 \mathrm{ml}$ of blood sample is collected in nonheparinised serum bottle and sent for thyroid profile test. Components of thyroid profile included in our study

$>$ Serum Triiodothyronine (T3)

$>$ Serum thyroxine (T4)

$>$ Serum thyroid stimulating hormone (TSH)

\section{Result}

In our Study 50 Patient of CKD admitted were studied, out of which 37 (74\%) were with Haemodialysis and 13 (26\%) were without Haemodialysis.

In our Study 50 Patient of CKD who were on conservative management fulfilling the criteria for CKD were studied, among these 41 were males and 9 were females, their age varied from 15 to 75 Years.
Among the 50 patients in our study, patients who were 25 year old and below were 7 constituting 14\%, between 26 60 years were 38 constituting $78 \%$ and above 60 years of age were 5 in number constituting $10 \%$.

Out of the patients, 33 patients had GFR of less than 15 $\mathrm{ml} /$ minute accounting to $66 \%, 15$ patients had GFR ranging from $15-30 \mathrm{ml} /$ minute accounting for $30 \%$ and 2 patients had GFR ranging from more than $30 \mathrm{ml} /$ minute accounting for $4 \%$. Among the patient studied most were in the range of creatinine clearance $<15 \mathrm{ml} /$ minute.

In our study out of 50 patient, 33 patients had low serum T3 levels (66\%), 4 patients among low serum T3 value, they also had low T4 and high TSH suggesting primary hypothyroidism (8\%). So excluding 4 patients of hypothyroidism 29 patients had low T3 syndrome in our study.

16 patients had low T4 levels in our study, out of which 4 patients had low T3 and high TSH suggesting primary hypothyroidism. Excluding hypothyroidism 12 (24\%) patients had low T4 in our study.

The TSH values in our study ranged from $0.6-8 \mathrm{micro} \mathrm{IU} / \mathrm{ml}$. Among 50 patients, 46 patients were in the normal range and 4 patients had high value of more than 20 micro IU/ ml. In patients who were in the high range 3 were males and 1 was female.

Out of 50 patients in our study 34 patients (68\%) had the symptoms suggestive of hypothyroidism such as tiredness, weakness, cold intolerance, dry coarse skin, constipation, hoarseness of voice, loss of hair, etc. 
Out of 29 patients who had low T3 syndrome, 19 patients had symptoms suggestive of hypothyroidism accounting for $65.5 \%$ and 4 patients among the primary hypothyroidism, all four had symptoms of hypothyroidism which accounts for $100 \%$.

Among 50 patients of CKD, 17 patients did not show any thyroid function abnormalities but out of them 11 had symptoms suggestive of hypothyroidism which accounts for $64.7 \%$.

Features of hypothyroidism such as delayed ankle jerk was present in 2 patients, out of which one were hypothyroid. Wound was found in one patient who is a hypothyroid. 24 hours urinary protein excretion was $<1 \mathrm{~g} /$ day in all the patients in our study.

Out of 50 patients in our study, 48 patients had anaemia, out of which 2 male and 2 female patients are in normal range

\section{Conclusion}

$>$ In our study population, 50 CKD patients who were on conservative management were studied. Among them $68 \%$ of the patients had low T3 values.
$>$ The change in the serum levels of T3 and T4 in patients with CKD can be considered as being protective, promoting conservation of protein.

$>$ There is progressive increase in the number of patients with Low T3 and T4 syndrome with the severity of renal failure.

$>$ There is increase in incidence of hypothyroidism in patients with chronic kidney disease.

$>$ Excluding hypothyroidism T3 level is found to be low in $58 \%$ of the patients and T4 level is low in $24 \%$ of the patients. .

$>$ As the age increases there is increase in incidence of Low T3 syndrome in patients with CKD.

$>$ In patients with low GFR the serum T3 level was found to be decreased. This shows a direct linear relationship between GFR and T3 level.

\section{Limitations of This Study}

Thyroid dysfunction was studied in patients with CKD irrespective of the etiology of CKD therefore individual correlation of the etiology of CKD with thyroid dysfunction could not be studied. Thyroid dysfunction was not studied in patients on dialysis, as dialysis itself affects the thyroid profile independently of CKD.

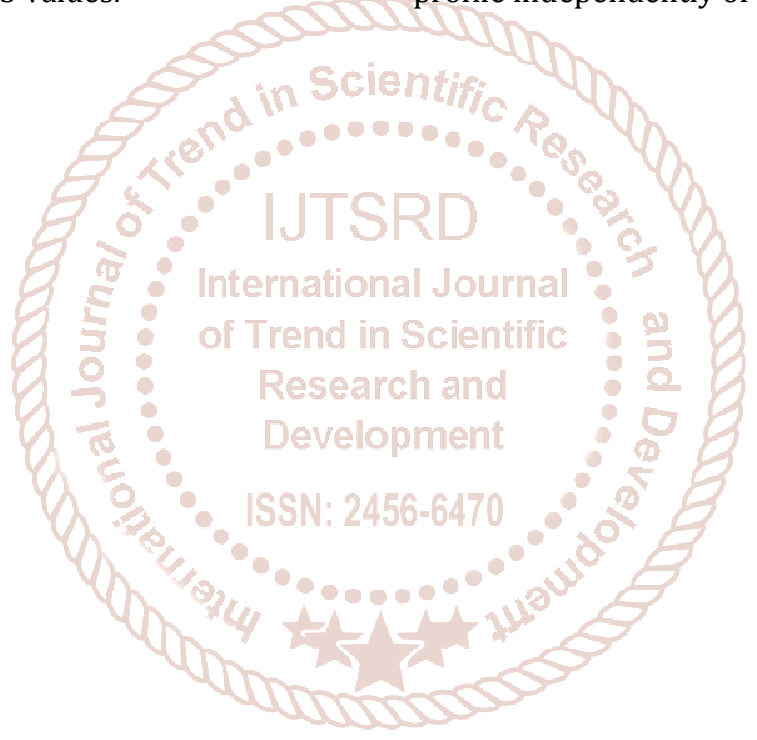

